\title{
Crystal structure of the $\mathrm{Al}_{8} \mathrm{Cr}_{5}$-type intermetallic $\mathrm{Al}_{7.85} \mathrm{Cr}_{5.16}$
}

\section{Xu Geng, Bin Wen and Changzeng Fan*}

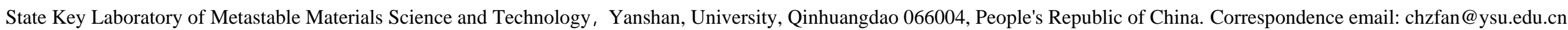

Supplementary materials include:

Table S1 Crystallographic information of all reported $\mathrm{Al}_{8} \mathrm{Cr}_{5}$ phases

Table S2. Lattice parameters for the standardization of crystal data for the $\gamma_{2}{ }^{\prime}-\mathrm{Al}_{8} \mathrm{Cr}_{5}$ phase based on the refined crystal data

Table S3. The atomic positions for the standardization of crystal data for the $\gamma_{2}{ }^{\prime}-\mathrm{Al}_{8} \mathrm{Cr}_{5}$ phase based on the refined crystal data 
Table S1 Crystallographic information of all reported $\mathrm{Al}_{8} \mathrm{Cr}_{5}$ phases

\begin{tabular}{|c|c|c|c|c|c|c|c|c|c|c|}
\hline \multirow{3}{*}{$\begin{array}{l}\text { Phase } \\
\mathrm{Al}_{8} \mathrm{Cr}_{5}\end{array}$} & \multicolumn{2}{|c|}{ Space Group } & \multicolumn{2}{|c|}{ Lattice Parameters } & \multicolumn{5}{|c|}{ Wyckoff positions } & \multirow[t]{2}{*}{ Ref. } \\
\hline & \multirow{2}{*}{$\begin{array}{l}\text { Symbol } \\
I-43 m\end{array}$} & \multirow{2}{*}{$\begin{array}{l}\text { number } \\
217\end{array}$} & \multirow{2}{*}{$\begin{array}{l}a(\AA) \\
9.0900\end{array}$} & \multirow{2}{*}{$\begin{array}{l}\alpha\left(^{\circ}\right) \\
90.0000\end{array}$} & \multicolumn{2}{|c|}{ Atom site } & \multirow{2}{*}{$\begin{array}{l}x \\
0.3087\end{array}$} & \multirow{2}{*}{$\begin{array}{l}y \\
0.3087\end{array}$} & \multirow{2}{*}{$\begin{array}{l}z \\
0.0394\end{array}$} & \\
\hline & & & & & Al1 & $24 g$ & & & & {$[1]$} \\
\hline \multirow[t]{5}{*}{ (1992) } & & & & & $\mathrm{A} 12$ & $8 c$ & 0.1062 & 0.1062 & 0.1062 & \\
\hline & & & & & $\mathrm{Cr} 1$ & $8 c$ & 0.1062 & 0.1062 & 0.1062 & \\
\hline & & & & & Al3 & $12 e$ & 0.3522 & 0 & 0 & \\
\hline & & & & & $\mathrm{Cr} 2$ & $12 e$ & 0.3522 & 0 & 0 & \\
\hline & & & & & $\mathrm{Cr} 3$ & $8 c$ & 0.8300 & 0.8300 & 0.8300 & \\
\hline $\mathrm{Al}_{16} \mathrm{Cr}_{9.5}$ & $R 3 m$ & 160 & 7.8110 & 109.1300 & Al1 & $3 b$ & -0.3608 & -0.3608 & -0.0095 & {$[2]$} \\
\hline \multirow[t]{10}{*}{ (1977) } & & & & & $\mathrm{A} 12$ & $3 b$ & 0.0055 & 0.0055 & -0.2096 & \\
\hline & & & & & $\mathrm{A} 13$ & $3 b$ & -0.2709 & -0.2709 & -0.6270 & \\
\hline & & & & & $\mathrm{A} 14$ & $3 b$ & 0.3699 & 0.3699 & 0.0306 & \\
\hline & & & & & A15 & $6 c$ & -0.3179 & 0.2982 & 0.0533 & \\
\hline & & & & & Al6 & $3 b$ & 0.3608 & 0.3608 & 0.5720 & \\
\hline & & & & & $\mathrm{Cr} 1$ & $1 a$ & 0.1795 & 0.1795 & 0.1795 & \\
\hline & & & & & $\mathrm{Cr} 2$ & $3 b$ & 0.0055 & 0.0055 & -0.2096 & \\
\hline & & & & & $\mathrm{Cr} 3$ & $1 a$ & -0.3071 & -0.3071 & -0.3071 & \\
\hline & & & & & $\mathrm{Cr} 4$ & $3 b$ & 0.3699 & 0.3699 & 0.0306 & \\
\hline & & & & & $\mathrm{Cr} 5$ & $3 b$ & 0 & 0 & 0.3451 & \\
\hline
\end{tabular}




\begin{tabular}{lllllllll}
$\mathrm{Al}_{8} \mathrm{Cr}_{5} \quad \mathrm{R3m} \quad 160$ & 7.8050 & 109.1270 & $\mathrm{Cr} 1$ & $1 a$ & 0.1940 & 0.1940 & 0.1940 & {$[3]$} \\
$(1937)$ & $\mathrm{Cr} 2$ & $3 b$ & 0.0030 & 0.0030 & 0.7940 \\
& & $\mathrm{Cr} 3$ & $3 b$ & 0.9980 & 0.9980 & 0.3400 \\
& $\mathrm{Cr} 4$ & $3 b$ & 0.3550 & 0.3550 & 0.0060 \\
& $\mathrm{Al}$ & $1 a$ & 0.6720 & 0.6720 & 0.6720 \\
& $\mathrm{Al} 2$ & $3 b$ & 0.6540 & 0.6540 & 0.0120 \\
& $\mathrm{Al}$ & $3 b$ & 0.3490 & 0.3490 & 0.5820 \\
& $\mathrm{Al} 14$ & $3 b$ & 0.7220 & 0.7220 & 0.3560 \\
& $\mathrm{Al}$ & $6 c$ & 0.0330 & 0.2880 & 0.6610 \\
\hline
\end{tabular}

Table S2. Lattice parameters for the standardization of crystal data for the $\gamma_{2}{ }^{\prime}-\mathrm{Al}_{8} \mathrm{Cr}_{5}$ phase based on the refined crystal data

\begin{tabular}{ll}
\hline Chemical Formula & $\mathrm{Al}_{8} \mathbf{C r}_{5}$ \\
\hline Crystal system, space group & Hexagonal, $R 3 m$ \\
$a, b, c / \AA$ & $12.8717,12.8717,7.8408$ \\
$\alpha, \beta, \gamma / \circ$ & $90,90,120$ \\
$V / \AA^{3}$ & 1125.03 \\
\hline
\end{tabular}


Table S3. The atomic positions for the standardization of crystal data for the $\gamma_{2}{ }^{\prime}-\mathrm{Al}_{8} \mathrm{Cr}_{5}$ phase based on the refined crystal data

\begin{tabular}{|c|c|c|c|c|c|c|}
\hline Label & Site & $x$ & $y$ & $z$ & $U_{\text {iso }} * / U_{\text {eq }}$ & Occ. $(<1)$ \\
\hline Al1 & $9 b$ & $0.5977(12)$ & $0.4023(12)$ & $0.0591(9)$ & 0.010871 & 0.7720 \\
\hline Cr1 & $9 b$ & $0.5977(12)$ & $0.4023(12)$ & $0.0591(9)$ & 0.010871 & 0.2280 \\
\hline Al2 & $9 b$ & $0.2150(11)$ & $0.7850(10)$ & $0.0770(7)$ & 0.011734 & 0.5000 \\
\hline $\mathrm{Cr} 2$ & $9 b$ & $0.2150(11)$ & $0.7850(10)$ & $0.0770(7)$ & 0.011734 & 0.5000 \\
\hline Al3 & $9 b$ & $0.0917(14)$ & $0.9083(13)$ & $0.2161(9)$ & 0.012218 & 0.9580 \\
\hline Cr3 & $9 b$ & $0.0917(14)$ & $0.9083(13)$ & $0.2161(9)$ & 0.012218 & 0.0420 \\
\hline Cr4 & $3 a$ & $0.0000(10)$ & $0.0000(10)$ & $0.0000(7)$ & 0.005509 & \\
\hline Cr5 & $9 b$ & $0.4444(7)$ & $0.5556(7)$ & $0.2062(5)$ & 0.003182 & \\
\hline Cr6 & $9 b$ & $0.7846(8)$ & $0.2154(7)$ & $0.2256(6)$ & 0.011041 & \\
\hline Cr7 & $3 a$ & $0.0000(11)$ & $0.0000(11)$ & $0.4368(8)$ & 0.009053 & \\
\hline Al4 & $18 c$ & $0.3732(14)$ & $0.0161(14)$ & $0.0334(10)$ & 0.018686 & \\
\hline Al5 & $9 b$ & $0.5501(15)$ & $0.4499(16)$ & $0.3699(11)$ & 0.014880 & \\
\hline
\end{tabular}

[1] Braun, J., Ellner, M. \& Predel, B. (1992). J. Alloys Compd.183, 444-448.

[2] Brandon, J. K., Pearson, W. B., Riley, P. W., Chieh, C. \& Stokhuyzen, R. (1977). Acta Crystallogr. B.33, $1088-1095$.

[3] Bradley, A. J. \& Lu, S. S. (1937). Z. Kristallogr. Cryst. Mater.96, 20-37. 